This is a copy of the Accepted Manuscript and is not the version of record. The full version can be found at http://dx.doi.org/10.7748/nop.2017.e986 


\title{
Factors that influence older people's engagement with digital health technology
}

\author{
Authors: \\ Dr Katherine Curtis (Associate Professor and Head of Department Nursing and Clinical \\ Sciences) and \\ Kyle Price (BU Sept.2015 Cohort Physiotherapy Student)
}

\begin{abstract}
Digital Health Technology (DHT) encompasses a wide range of applications and interventions with the potential to address health needs within an increasingly ageing population. Older people's engagement with DHT depends on many factors and this commentary summarizes current understanding of the barriers and facilitators to DHT uptake and continued use among older people. It is evident that older people's confidence in using digital technology, their perceptions of personally benefiting from $\mathrm{DHT}$, its design, and the available support from health professionals and carers with using DHT, all influence engagement. Understanding these barriers and facilitators among the older population creates opportunities and solutions to enable greater numbers of older people to benefit from DHT. This paper provides information to those who work with older people and design digital health interventions for older people, to consider and address factors that influence their engagement with these important and rapidly evolving health and care innovations.
\end{abstract}

\section{Key words}

Digital Health, Technology, Older People, Barriers, Facilitators

\section{Introduction}

There are an increasing number of older people in the United Kingdom (UK) with health and well-being needs. The population of over 60 's in the UK is estimated to increase $20 \%$ by 2024 (Office of National Statistics 2014) with an associated increase in the numbers of older people living with long-term physical and mental health conditions such as cardiac failure and dementia, and with increasing frailty (Age UK 2017).

Digital health technology (DHT) is evolving rapidly in order to address some of these needs within the older population. DHT is a broad term that includes telecare such as mobile phone based digital communication as well as ambient monitoring devices such as wearable technology that measures mobility and risk of falling. Such technology is linked to desktop computers, tablets and/or smart phones as well as bespoke devices, and health and wellbeing information can be recorded or simultaneously communicated to enable health professionals to provide individualised advice or care. Examples of this are seen within personal alarms that link to emergency services and GPS monitoring for those who may be at risk due to wandering; thereby improving speed of access when urgent help is required for older people and when managing an individual's safety. DHT can assist people to live well, providing more autonomy in accessing health and social care staff when they require advice or assistance. It can better enable them to share in decision making about activities designed to meet their well-being and health needs. 
This rapidly changing digital healthcare environment is important to understand in relation to the potential benefit for older people. This article explores current understanding of some of the key facilitators and barriers for older people in their initial uptake and engagement with DHT and in their ongoing use of DHT once engaged.

\section{Facilitators and barriers to Initial DHT uptake and engagement}

\section{Perceived benefits of DHT introduction}

One specific influence on initial DHT uptake is older people's perception of the benefit they will gain from using DHT. The two most frequently identified uses of DHT among older people are related to better management of long-term conditions and improved safety at home.

In a study that required older people with rheumatoid arthritis (RA) to use smartphones to monitor their symptoms, users were highly motivated to participate because they expressed a perception that they would personally benefit alongside providing researchers with clinically relevant information (Reade et al. 2017). Middlemass et al. (2017) identified that older people with chronic obstructive pulmonary disease perceived an advantage of engagement with DHT through the monitoring of their health trends which enabled detection of the early onset of infections. In another study by Currie et al (2015), older people with chronic pain cited their reduced need to travel for treatment as a perceived benefit of engagement with DHT. These people lived in rural areas but were able to access a number of specialist pain management staff and participate in group sessions via a web-based applications, providing benefits that they may have had difficulty in accessing without DHT (Currie et al. 2015).

In relation to improved safety at home, ambient assisted living technology such as mobility monitoring devices, are seen by users as DHT that permits them to remain at home for longer and gives them confidence to safely access areas of their homes that were previously inaccessible (Hamblin 2017). This encourages initial engagement. Older people also identify DHT as having potential to help them to remain at home by managing communications in relation to falls. Williams et al. (2013) identified through focus-group discussion that wristworn falls sensors reassured older people in a way that enabled greater independence.

\section{The impact of the timing of DHT introduction to engagement}

A facilitator of DHT uptake among older people is the perceived positive impact it can have and the timing of DHT introduction is seen as an important factor in this perception. This is particularly important for helping self-management of a condition soon after initial diagnosis. For example, some DHT users identified that the self-care information and techniques within web-based programmes represented good encouragement for engagement when newly diagnosed with osteoarthritis (Algeo et al. 2017) and with angina (Devi et al. 2014). Current and potential users of DHT also feel that introduction of DHT immediately after a significant event such as hospital discharge or surgery would increase its effectiveness (Holliday et al. 2015).

However, it is also important to acknowledge that there are some prevailing attitudes among older people that indicate severity of illness and age are factors in engaging with DHT, with some feeling people need to have a high level of need or be "ill enough" to warrant use of DHT devices (Sanders et al. 2012; Doyle et al. 2014; Lie et al. 2016) and that certain interventions such as web-based programmes are for younger age groups (Currie et al. 
2015). Such perceptions are important to consider and manage if DHT is to be encouraged among older people.

\section{Competency and confidence with digital technology}

Another factor influencing DHT uptake is older people's competency and confidence in using existing technology and novel devices and this can be influenced through collaborative design with older people and provision of guidance and education on DHT. Older users' understanding of the function of devices such as modems and routers can influence their engagement, particularly with home telehealth equipment such as online applications provided on an iPad or smart phone (Sanders et al. 2012; Doyle et al. 2014). However, more recent studies suggest that a lack of prior technological knowledge is not a barrier to DHT uptake and it is the design that is the important factor (Middlemass et al. 2017), with older users preferring simple user interfaces (Doyle et al. 2014), and preferring avoidance of excessive text in large blocks and medical jargon (Algeo et al. 2017). Co-creation of the DHT interface with older users can improve uptake success, particularly when developing electronic assisted living technologies (Holliday et al. 2014).

The design and marketing of DHT products is also important to consider in relation to encouraging uptake. Holliday et al. (2015) identified through focus groups with older people that their self-image was affected if DHT products appeared to label users as frail or disabled. Where potential users have co-designed DHT products, such as wearable fall sensors, they reported not feeling stigmatised when wearing the device in public, suggesting uptake may be encouraged through a co-creation approach with older people (Williams et al. 2013, Holliday et al. 2015).

These issues related to upholding dignity were identified alongside other issues related to the trustworthiness of the technology and its ability to maintain users' privacy. Older people are concerned about the nature of data that is collected by DHT, that the technology might record aspects of their lives that they have not consented to sharing, and they want confirmation that this isn't going to happen before use (Doyle et al. 2014). This anxiety and distrust barrier can be reduced by clearly explaining the purpose of the DHT devices in person, a strategy shown to reduce user anxieties about this issue (Doyle et al. 2014). Users also want to understand how their data is presented to others and have control over this (Lie et al. 2016).

One further area that older people consider important in their decision to adopt DHT is funding. Cost is less of an issue if users feel the product would benefit them and potentially less of a concern for 50-70 year olds who may still be working (Holliday et al. 2015). The literature demonstrates that DHT uptake by older people is therefore strongly influenced by perceived benefit, timing of introduction, design features, impact on dignity, and cost.

\section{Facilitators and barriers to continued use of DHT following initial engagement}

\section{Re-evaluating ongoing benefit}

The continued use of DHT by older people relies upon their re-evaluation of the ongoing benefits, how the design enables varied and interesting ways to engage with the technology, and the impact of it on the users' behaviour and the users' relationships with those around them. Some literature identifies that difficulties in its use following uptake challenge the perception of ongoing benefit from the DHT and thereby can result in discontinuation of its use. For example, some users of DHT containing global positioning systems that monitor the 
whereabouts of the user had a negative experience due to the poor battery life of the device because its function ceased unless the battery was frequently recharged. The need for frequent battery charging can become an obstacle to the free movement of the person using the DHT and hence resulted in restricting their freedom rather than increasing it (Hamblin 2017). Such experiences could be avoided with improved technology and co-created design with the end users' needs in mind.

Another challenge that restricts the continued use of adopted DHT is poor integration of it with the users' existing technology. For example, older users of smart phone applications who did not have regular or reliable access to Wi-Fi or owned older devices found that the technology was too slow or that memory storage was too small for the DHT to be of any real value (Reade et al. 2016). Users also had less intention to continue their use of DHT when they experienced malfunctions (Reade et al. 2016, Hamblin 2017) even when some of these were related to them forgetting to charge a device. However, Doyle et al. (2014) found that this potentially negative experience in DHT use among older people could be overcome through pro-activity in the design features and support service. Their devices reminded users when to replace batteries, components to be swapped or fixed remotely via an internet connection, and they had fast technical help response times when users identified problems. However, poor internet connection was an ongoing barrier to DHT for older people living in rural locations due to slower download speeds and inability to use videoconferencing software. It is important to consider the appropriateness of DHT design that relies on webbased services when identifying potential users living outside urban areas (Currie et al. 2015).

Experiences such as these, where the technology did not live up to users' initial expectations, can prevent continued use. However, literature identifies some very successful DHT introductions that encouraged ongoing use. One example is the study by Williams et al. (2014) using a tablet-computer based intervention for people with chronic obstructive pulmonary disease (COPD). This DHT resource supported self-management and users reported that it helped them to feel more in control of their condition when interviewed 6 months after commencing use of the DHT. These users also reported having a better understanding of what their oxygen saturations should be and less need for reassurance from healthcare professionals (Williams et al. 2014). Another example of successful ongoing use is the website-based dizziness management programme reported by Essery et al. (2017). Users noticed improvements in their symptoms which motivated them to continue with an exercise programme, despite the challenging nature of the exercises.

\section{Behaviour change and self management}

Continued use of DHT can influence positive behaviour change and is recognised as a behaviour change techniques by the National Institute for Health and Care Excellence (NICE) (2014). A number of smart phone based applications in the NHS apps library include behaviour change focussed technology (McMillan et al. 2016). Although these are most commonly aimed at changing unhealthy behaviours in people without long-term conditions, a number of these DHT interventions include behaviour change techniques that enable users to set individualised goals (Devi et al. 2014, Algeo et al. 2017) and encourage them to monitor their progress using diaries, such as recording the amount of exercise they take or trends in symptoms (Devi et al. 2014, Williams et al. 2014). Exercise-based interventions can give positive feedback at regular intervals and reassure or inform users of their progress and thereby encourage ongoing use (Doyle et al. 2014, Essery et al. 2016). The literature shows that a perceived lack of feedback is viewed negatively by users of DHT, such as within the studies for people using DHT for managing their respiratory or heart disease, and 
these users expressed that they who would have preferred input on their progress from medical staff (Middlemass et al. 2017).

\section{Support and peer networks}

Another factor influencing continued use of DHT is social support such as through peer networks. For example, users of a smart phone application to track rheumatoid arthritis symptoms suggested that access to an online forum would have helped to maintain their engagement; however this may reflect the needs of a relatively younger group of participants (Reade et al. 2016). A website for dizziness management included "Retraining Stories" as a means of sharing others' experiences of the intervention (Essery et al. 2017) and could be considered as a form of social or peer support. Other DHT applications included the facility to contact a cardiac nurse via email or a scheduled chat room session (Devi et al. 2014) and thereby provided a social and professional support network.

Despite the potential for DHT to increase older people's autonomy in managing their health, the involvement of relatives and carers and support from healthcare professionals are seen as influencing the continued use of DHT. Older people with long term conditions such as COPD and heart disease feel reassured that DHT allows healthcare professionals to monitor their condition and intervene if necessary (Middlemass et al. 2017). However, it is also important to consider that a balance is needed between DHT and direct human involvement with the user, in that DHT could reduce the perception of support received through contact with monitoring staff or if a person that the older person was not expecting attended their home in response to an alert triggered by their DHT (Hamblin 2017). These examples suggest that a lack of integration between older peoples' usual sources of care and the DHT could be a reason for some to withdraw from a DHT study and discontinue DHT use (Sanders et al. 2012). DHT could also be perceived as disrupting established routines that users relied on such as check-in calls by relatives or be perceived as a threat to in-person visits by carers and healthcare staff (Sanders et al. 2012, Lie et al. 2016).

One further factor that encourages continued use of DHT is the provision of technical support (Reade et al. 2016). Doyle et al. (2014) also suggest that older people can be afraid of making mistakes with new technology and noted that study participants often relied on telephone support lines simply to get reassurance that they had not broken the equipment. This suggests that at the very least, sensitive technical support for older people's use of DHT helps with their experience of using new technology and if it goes smoothly they are more likely to continue their engagement.

\section{The novelty factor}

In a number of studies run by the Technology Research for Independent Living (TRIL) centre, researchers found that users' motivation to engage with DHT decreased over time, which they attributed to boredom or lack of novelty (Doyle et al.2014). Some DHT applications and web sites address this by including interactive components such as quizzes, case studies and informative pictures and videos that enhance learning and are updated regularly (Currie et al. 2015, Algeo et al. 2017) and these can encourage older people's engagement. Essery et al. (2017) found that almost all users who completed a trial of the dizziness management website felt it offered new information and taught new skills, despite some of the users having already completed the same activity using a booklet-based format (Essery et al. 2017). 


\section{Conclusions and implications for practice}

Through this exploration of facilitators and barriers for DHT use by older people, it is clear there remain opportunities to improve engagement at both the uptake phase and in the continued use of DHT. One opportunity is within the design phase. Improved collaboration and consulting with older people by design technologists to co-create DHT user interfaces can improve their engagement with DHT, as it ensures usability and will result in greater confidence in the technology among this population. The available resources in the older person's environment should also be considered in the design as applications that require uninterrupted internet connections or high download speeds may not function as intended in different areas of the country.

The timing of DHT introduction is also a key factor, in that the nearer to initial diagnosis or need identification the better for recognition of potential benefit by older users. The implications for practice are that DHT opportunities are identified and offered as early as possible to those who could benefit.

Barriers to uptake can occur when older people are first introduced to DHT or arise through their continued usage, due to a perception of a lack of support or low confidence. Providing reliable sources of technical, professional and social support, as well as preparatory education, during DHT introduction and ongoing provision needs to be considered by practice in order to encourage uptake and maintain engagement. Users also need to be reassured that $\mathrm{DHT}$ is trustworthy and will not negatively impact upon their dignity.

Reassurance that DHT use does not mean a complete removal of other health or care support services is also important in managing concerns.

Enabling older people to engage with DHT has the potential to improve their health and wellbeing. By addressing the facilitators and barriers identified in this commentary article, healthcare practitioners and DHT designers can work more effectively alongside older people and start to maximise the potential benefit from this innovative technology.

\section{References}

AgeUK, 2017. Briefing: health and care of older people in England 2017. London, AgeUK.

Algeo N, Hunter D, Cahill A, et al (2017) Usability of a digital self-management website for people with osteoarthritis: A UK patient and public involvement study. International Journal of Therapy and Rehabilitation. 24, 2, 78-82.

Currie M, Philip L, Roberts, A (2015) Attitudes towards the use and acceptance of ehealth technologies: a case study of older adults living with chronic pain and implications for rural healthcare. BMC Health Services Research. 15, 162, 1-12.

Devi R, Carpenter, C, Powell J et al (2014) Exploring the experience of using a web-based cardiac rehabilitation programme in a primary care angina population: A qualitative study. International Journal of Therapy and Rehabilitation. 21, 9, 434-440.

Doyle J, Bailey C, Scanaill C et al (2014) Lessons learned in deploying independent living technologies to older adults' homes. Universal Access in the information society. 13, 191204.

Essery R, Kirby S, Geraghty A et al (2017) Older adults' experiences of internet-based vestibular rehabilitation for dizziness: A longitudinal study. Psychology and Health. 1-17. 
Hamblin K, (2017) Telecare, obtrusiveness, acceptance and use: An empirical exploration. British Journal of Occupational Therapy. 80, 2, 132-138.

Holliday N, Ward G, Fielden S (2015) Understanding younger older consumers' needs in a changing healthcare market - supporting and developing the consumer market for electronic assisted living technologies. International Journal of Consumer studies. 39, 305-315.

Lie M, Lindsay S, Brittain K (2016) Technology and trust: older people's perspectives of a home monitoring system. Ageing and Society. 1501-1525.

McMillan B, Hickey E, Patel M et al (2016) Quality assessment of a sample of mobile appbased health change interventions using a tool based on the National Institute of Health and Care Excellence behaviour change guidance. Patient Education and Counselling. 99, 429435.

Middlemass J, Vos J, Siriwardena A (2017) Perceptions on use of home telemonitoring in patients with long term conditions - concordance with the Health Information Technology Acceptance Model: a qualitative collective case study. BMC Medical Informatics and Decision Making. 17, 89, 1-13.

National Institute for Health and Care Excellence (NICE) (2014) Behaviour Change: Individual Approaches. London, NICE.

Office for National Statistics (2015) National Population Projections: 2014-based Statistical Bulletin. London, Office for National Statistics.

Reade S, Spencer K, Sergeant J et al (2017) Cloudy with a chance of pain: engagement and subsequent attrition of daily data entry in a smartphone pilot study tracking weather, disease severity, and physical activity in patients with rheumatoid arthritis. Journal of Medical Internet Research Mhealth and Uhealth. 5, 3, 1-12.

Sanders C, Rogers A, Bowen R et al (2012) Exploring barriers to participation and adoption of telehealth and telecare within the Whole System Demonstrator trial: a qualitative study. BMC Health Services Research. 12 ,220, 1-12.

Williams V, Victor C, McCrindle R (2013) It is always on your mind: Experiences and perceptions of falling of older people and their carers and the potential of a mobile falls detection device. Current Gerontology and Geriatrics Research. 1-7.

Williams V, Price J, Hardinge M et al (2014) Using a mobile health application to support self-management of COPD. British Journal of General Practice. e392-e400. 